\title{
Utilizing guided acoustic waves to measure dispersive material properties of polymers
}

\author{
Jens Rautenberg ${ }^{1}$, Fabian Bause ${ }^{1}$, Bernd Henning ${ }^{1}$ \\ ${ }^{1}$ Measurement Engineering Group, University of Paderborn, \\ Warburger Str. 100, Paderborn, Germany \\ Rautenberg@emt.uni-paderborn.de
}

\begin{abstract}
:
This contribution will give a short introduction to the most important mechanic and acoustic parameters that are necessary to model and simulate frequency dependent sound propagation (dispersion) in isotropic but linear viscoelastic materials. Furthermore, several experimental techniques to measure these parameters will be discussed, like the dynamic-mechanical analysis and transient ultrasonic techniques. Finally it will be shown how to use the determined material parameters for the simulation of transient signals in a highly attenuative acoustic waveguide.
\end{abstract}

Key words: Material dispersion, guided waves, material characterization, DMA, linear viscoelasticity

\section{Introduction}

Ultrasonic measuring systems are well established in nondestructive testing and the evaluation of metal-like materials. Therein the material parameters are almost constant with frequency which is not valid for polymers any more. This is why quasi static methods (e.g. tensile tests) and ultrasonic tests result in different material parameters and standard inspection methods cannot be directly applied to fiber composites for instance. It is important to know the frequency dependent material behavior beforehand, and this leads to the aim of this work: The utilization of guided acoustic waves to measure all acoustically relevant material parameters at one preconditioned sample, in particular as a function of frequency.

The first part of the article will introduce a simple model to cope with linear viscoelasticity in the frequency domain. As a consequence of this model, material dispersion will be introduced as well. The next chapter will give a short survey about possible experimental techniques to measure the frequency dependent material parameters. The focus will be on the dynamic mechanical analysis and transient ultrasonic methods. The latter will be discussed in detail as it is the original part of the authors. As an example, Polyamide 6 (PA 6) was chosen, which is very sensitive towards frequency, even at room temperature. In addition, it has a high practical relevance, e.g. as a matrix material in organic composite sheets.

\section{Linear viscoelastic material model}

At low frequencies the considered polymers typically show delayed reactions to stepped strain or stress excitation. This viscoelastic behavior is quite universally represented by the fractional Zener model [1]:

$$
\sigma(t) \cdot\left(1+\tau_{\sigma}^{\beta} \frac{{ }_{\partial}^{\beta}}{{ }_{\partial t} \beta}\right)=E \cdot \varepsilon(t)\left(1+{ }_{\varepsilon}^{\beta} \frac{{ }_{\partial} \beta}{{ }_{\partial t} \beta}\right)
$$

The relationship between mechanical stress $\sigma$ and strain $\varepsilon$ is generally time dependent, but converges to the well-known Hooke's law with the modulus of elasticity $E$, if the relaxation times $\tau_{\sigma}, \tau_{\varepsilon}$ for stress and strain approximate zero - which is usually the case for metal-like materials. To ensure a nonnegative rate of energy dissipation and nonnegative internal work, it is necessary to constrain the model parameters [2]:

$$
E>0, \quad \tau_{\varepsilon} \geq \tau_{\sigma}>0, \quad 1 \geq \beta>0
$$

The fractional derivatives of order $\beta$ in eq. (1) can easily be treated in the Fourier domain. Therewith, it is possible to determine frequencydependent material properties, for example the stiffness matrix

$\mathbf{C}(\omega)=\mathbf{C} \cdot \frac{1+\left(j \omega \tau_{\varepsilon}\right)^{\beta}}{1+\left(j \omega \tau_{\sigma}\right)^{\beta}}$, 
wherein $\mathbf{C}$ is the real valued stiffness matrix of the not necessarily isotropic material and $\omega$ is the angular frequency [3].

\section{Dispersion in linear viscoelastic materials}

In such a time and frequency dependent material the sound velocity $c_{\mathrm{ph}}(\omega)$ and the absorption $\alpha(\omega)$ are frequency dependent, too:

$$
\begin{aligned}
& c_{\mathrm{ph}}(\omega)=c_{0} \cdot \mathfrak{R}\left\{\sqrt{\frac{1+\left(j \omega \tau_{\sigma}\right)^{\beta}}{1+\left(j \omega \tau_{\varepsilon}\right)^{\beta}}}\right\}^{-1} \\
& \alpha(\omega)=\frac{\omega}{c_{0}} \cdot \mathfrak{I}\left\{\sqrt{\frac{1+\left(j \omega \tau_{\sigma}\right)^{\beta}}{1+\left(j \omega \tau_{\varepsilon}\right)^{\beta}}}\right\}^{\beta}
\end{aligned}
$$

Herein, $c_{0}$ is the nonnegative sound velocity at very low frequencies for any kind of fundamental wave (longitudinal, transverse, etc.) [3]. Fig.1 exemplifies the frequency dependence of sound velocity and absorption for a typical polymer, Polypropylene (PP).

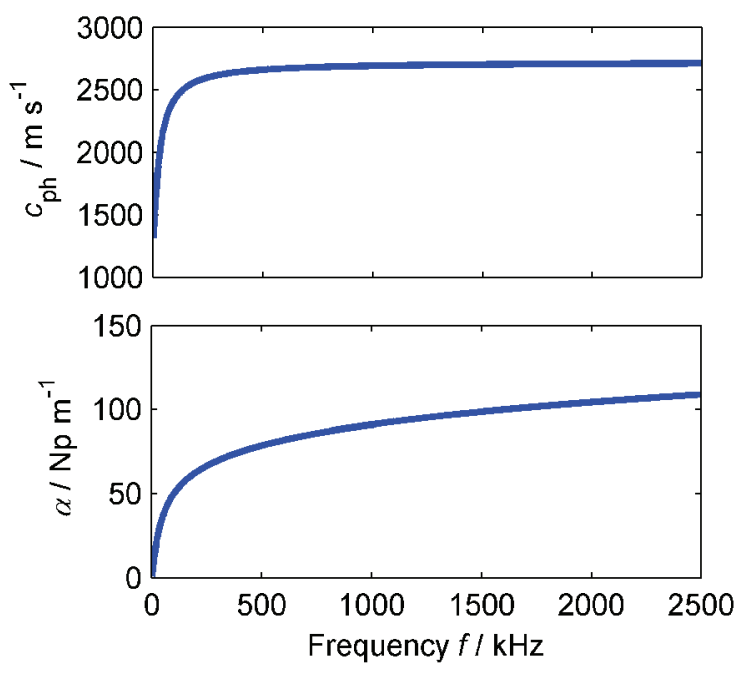

Fig. 1. Longitudinal sound velocity (top) and sound absorption (bottom) in a dispersive material (PP)

Due to the fact that the material model itself demands time causality, it can be shown that the sound velocity and attenuation given in eq. (4) and (5) also fulfill the Kramers-Kronig relationship which is sufficient for time causal modeling.

\section{Experimental techniques}

The mechanical properties can be determined in various ways. Depending on the frequency range of interest, the previously described mechanical models can be parameterized with quasi-static, low-frequency dynamic or high frequency ultrasound methods (see Fig. 2).

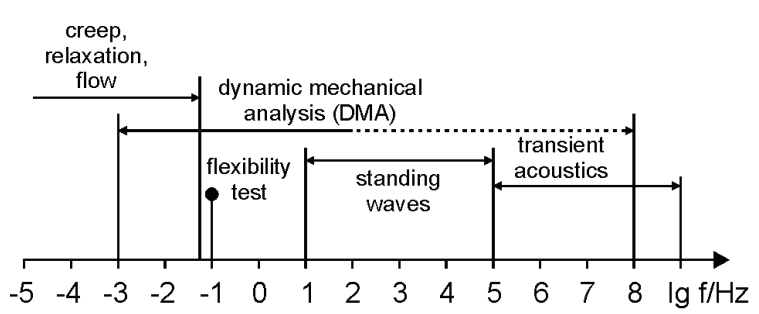

Fig. 2. Different experimental techniques for polymer characterization in a logarithmic frequency scale

To cover the whole frequency range, several methods may be combined [4]. For ultrasonic sensor devices a combination of transient acoustic measurements and dynamic mechanical analysis (DMA) may be satisfactory. In the following, the different methods will be discussed shortly.

\section{a) Quasi-static (tensile) testing}

Tensile tests, e.g. the stretching of a flat test specimen (see Fig. 3), can be applied to obtain stress-strain curves. Therewith, among others, the modulus of elasticity and Poisson's ratio can be determined.

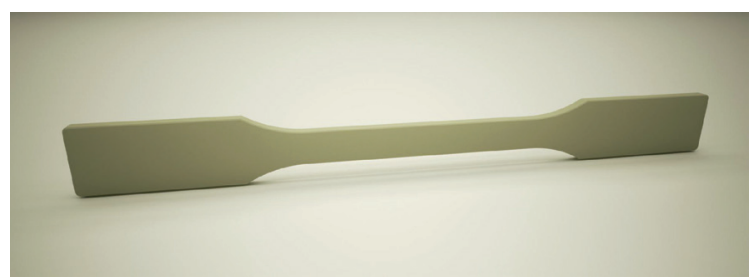

Fig. 3. Typical specimen (DIN EN ISO 527, Type $1 A$ and $1 B$ )

These parameters can be used to calculate longitudinal as well as transverse sound velocity. But the values are not suited to provide satisfactory parameters for ultrasound calculations in the high frequency range.

\section{b) Dynamic-mechanical analysis (DMA)}

The DMA (DIN EN ISO 6721) is an experimental technique to determine the complex modulus of elasticity. Therefor a clamped specimen is put under periodic stress. Stress $\sigma$ and strain $\varepsilon$ are measured simultaneously:

$$
\begin{aligned}
\sigma(t) & =\sigma_{0} \cdot \sin (\omega t+\delta) \\
& =\sigma_{0} \cdot(\sin (\omega t) \cos (\delta)+\cos (\omega t) \sin (\delta)) \\
\varepsilon(t) & =\varepsilon_{0} \cdot \sin (\omega t)
\end{aligned}
$$

The typical phase lag $\delta$ in viscoelastic materials is representative for the losses, which can be seen, if the time periodic signal parts in eq. (6) are reinterpreted as real and imaginary parts:

$$
\underline{E}(t)=\frac{\sigma(t)}{\varepsilon(t)}=\frac{\sigma_{0}}{\varepsilon_{0}} \cos (\delta)+j \frac{\sigma_{0}}{\varepsilon_{0}} \sin (\delta)
$$


The ratio of imaginary and real part of the complex modulus is also known as loss $\tan \delta$. By varying the temperature and operation frequency the so called time-temperature superposition can be utilized to extend the measurement range many times. Therein the results for different temperatures can shifted in a logarithmic time or frequency scale to obtain an overall master curve. Fig. 4 exemplifies the time-temperature superposition.

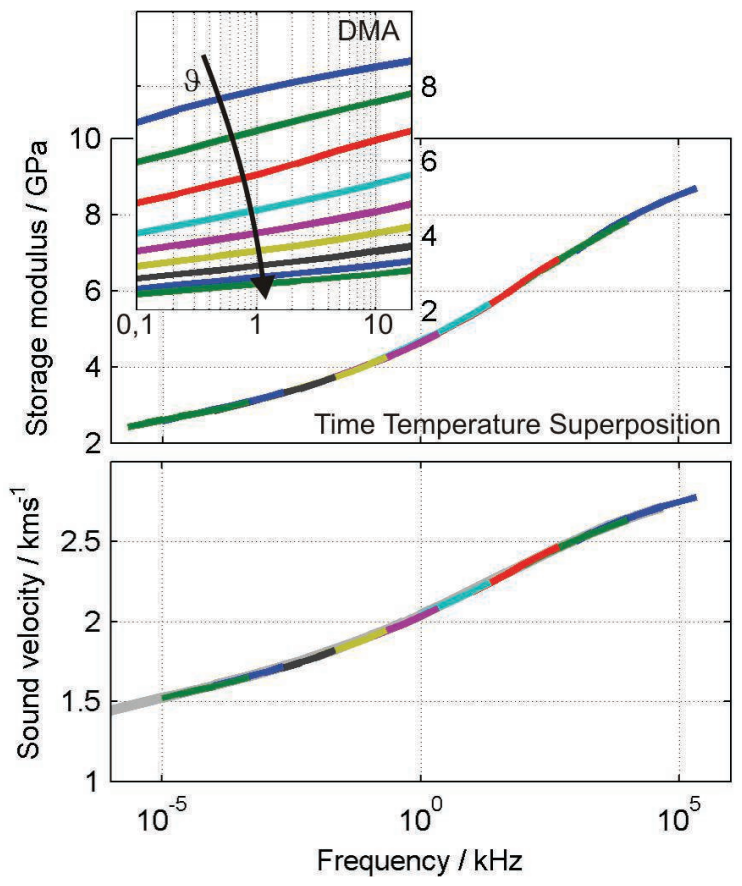

Fig. 4. Several steps of a DMA (top: original measured values; middle: combined values, using timetemperature superposition; bottom: deduced longitudinal sound velocity)

The high frequency extrapolations may be also a good starting point for ultrasonic calculations as the sound velocity $c$ can be calculated from complex modulus $\underline{E}$ and density $\rho$ (Fig. 4):

$c(\omega)=\Re\left\{(E(j \omega) / \rho)^{-0.5}\right\}^{-1}$

In a linear scale the sound velocity of Fig. 4 is very similar to the theoretical curve of Fig. 1:

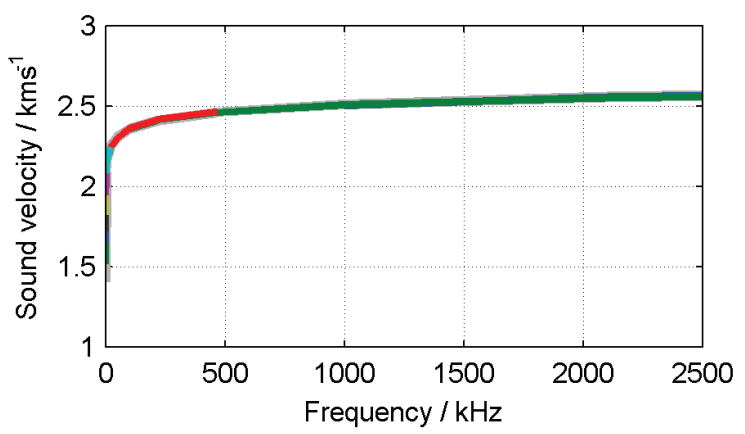

Fig. 5. Sound velocity measured with DMA and calculated with time-temperature superposition.
Only two of the measured DMA-curves (green and red) are necessary to map the typical frequency band of ultrasound applications. But these curves are far away from the original frequency band of the DMA and it is not clear, if the values are representative for ultrasonic applications [5]. Another problem is that the time-temperature superposition can only be applied to thermo-rheologic simple materials (e.g. PP or PA6 but not PEEK). A material's simplicity can be proved with a continuously curved Cole-Cole-diagram, which is the plot of loss over storage modulus [6].

The same procedure can then be done for the loss modulus and frequency dependent sound attenuation.

\section{c) Testing with bulk acoustic waves}

If a plate of small thickness but large lateral dimension should be investigated, the so-called immersion technique is a good choice. Plane acoustic waves in a liquid filled basin are used to measure the time of flight (TOF) between two ultrasound transducers with and without the specimen. At normal incident angle and with known thickness of the specimen, the velocity of the longitudinal wave can be calculated. At certain angles of incident it is also possible to excite transversal waves in the plate. If the plate is thick enough it is also possible to utilize the back-reflection technique with only one transducer. More sophisticated methods enable also to measure anisotropic $[7,8]$ or viscoelastic properties [9].

\section{d) Testing with guided acoustic waves}

The idea for the simultaneous determination of several acoustic parameters originates to the early 1950's [10]. The specimens were cylindrical with a large diameter in comparison to the used wavelength. Transmission measurements in axis through the rod show characteristic pulse trains:

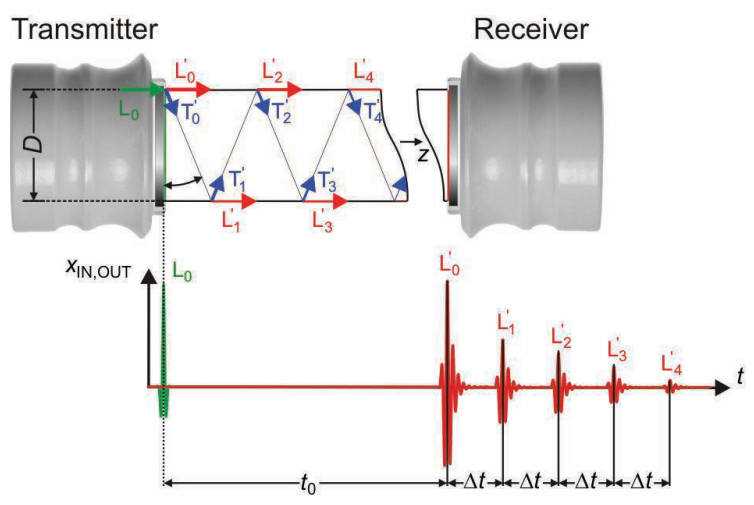

Fig. 6. Qualitative illustration of the measurement setup and the sending as well as the received signal 
The pulse arrival time $t_{0}$ as well as the time differences $\Delta t$ can be used to calculate the longitudinal and transversal wave speeds:

$c_{\mathrm{L}} \approx \frac{I}{t_{0}}, \quad c_{\mathrm{T}} \approx c_{\mathrm{L}} \cdot\left(1+\left(\frac{c_{\mathrm{L}} \cdot \Delta t}{D}\right)^{2}\right)^{-0,5}$

Due to the high sound absorption in polymers the size of the specimen must be significantly reduced in comparison to the original setup. Therewith the dispersion increases to such a degree that the assumed sound propagation rays loose validity and eq. (9) gets invalid, too.

This is why we use an inverse solution scheme: Based on a semi analytical model the received signal is simulated with assumed material parameters. These are iteratively changed until the simulated signal or its characteristic parameters are in good agreement with the measured ones.

\section{Model of sound propagation}

Both, the frequency dependent material dispersion (Fig. 1), and the geometric dispersion (see Fig. 7) have to be considered for the simulation of wave propagation in the specimen which is in fact an acoustic waveguide [11]. The chart shown in Fig. 7 is computed with the Scaled Boundary FEM described in [12], and already takes into account the frequency dependence of the real-valued stiffness matrix.

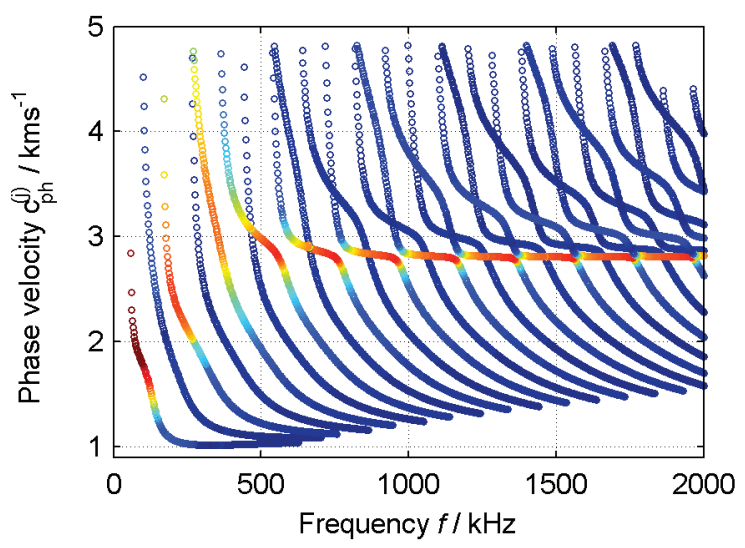

Fig. 7. Dispersion curves for a hollow cylindrical waveguide made of PA 6; the colors show from red to blue, to which extend the modes are excited with the chosen measurement setup (axial loading)

In our approach we succeeded in decomposing the complex frequency dependent elasticity matrix in a real valued frequency dependent elasticity matrix and the frequency dependent attenuation [3]. Therewith it is sufficient to use real valued parameters in the most relevant calculation steps and thereby it was possible to significantly reduce the computational effort for the inverse algorithm.
Taking also the shape of the propagative modes into account, it is possible to decompose any acoustic excitation into its modal components $A^{(i)}(j \omega)$. These components are the third dimension in Fig. 7 in terms of color. Due to an assumed axial loading mainly those modes are excited that propagate with nearly longitudinal sound velocity. The modal components can also be utilized to set up an acoustic transfer function $H(j \omega)$ :

$H(j \omega)=\sum_{i} A^{(i)}(\omega) \cdot \exp \left(\frac{j \omega l}{c_{\mathrm{ph}}^{(i)}(\omega)}\right)$

With an additional inverse Fourier transform it is possible to propagate an exciting signal through the waveguide to any other location $I$, even if the propagation media are attenuative.

\section{Results}

Both the DMA and the guided acoustic wave approach were tested with PA 6 specimens. On the one hand the DMA mastercurve was fitted with the fractional Zener model. On the other hand the parameters of the material model were modified to find the best match between measured and simulated signals at the end of the excited acoustic waveguide (a hollow cylinder: $I=16.9 \mathrm{~mm}$, inner and outer diameter are 6 and $18 \mathrm{~mm}$, respectively). The identified model parameters and the dispersion curves of the two experiments are completely different which was not expected on the basis of theory (see Fig. 8 and Tab. 1).
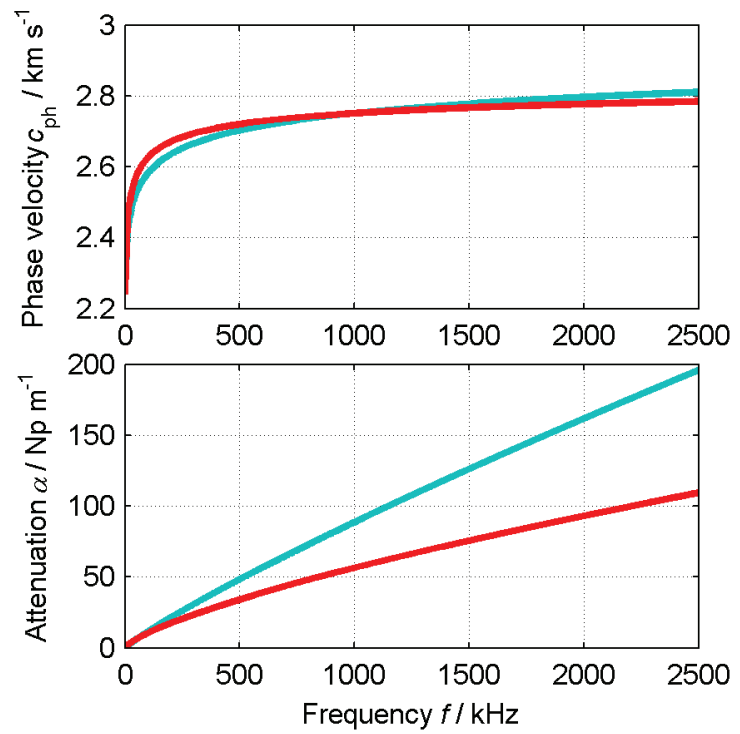

Fig. 8. Dispersion curves for PA 6, determined with DMA and time-temperature superposition (cyan) and guided ultrasonic waves (red).

Especially the attenuation is very different, whereas the sound velocity is comparable. 
Tab. 1: Identified material parameters

\begin{tabular}{|c|c|c|}
\cline { 2 - 3 } \multicolumn{1}{c|}{} & DMA & US \\
\hline$c_{0} / \mathrm{m} / \mathrm{s}$ & 822.5 & $1060\left(c_{\mathrm{L}}\right), 430\left(c_{\mathrm{T}}\right)$ \\
\hline$\tau_{\sigma} / \mathrm{ms}$ & 0.0021 & 0.1 \\
\hline$\tau_{\varepsilon} / \mathrm{ms}$ & 1.4035 & 79 \\
\hline$\beta$ & 0.21 & 0.3 \\
\hline
\end{tabular}

For the dispersion curves of the DMA data in Fig. 8 the quiescent sound velocity $c_{0}$ has to be adopted to the longitudinal value in Tab. 1 . This can be explained with the experiment setup of the DMA. In the acoustic experiment it is possible to distinguish between the elementary waves. In the DMA the exited mode always depends on all elastic constants and therewith must result in effective values.

Nevertheless, although the damping is very different, both model parameter sets are suited to simulate sound propagation in an attenuative acoustic waveguide (see Fig. 9).
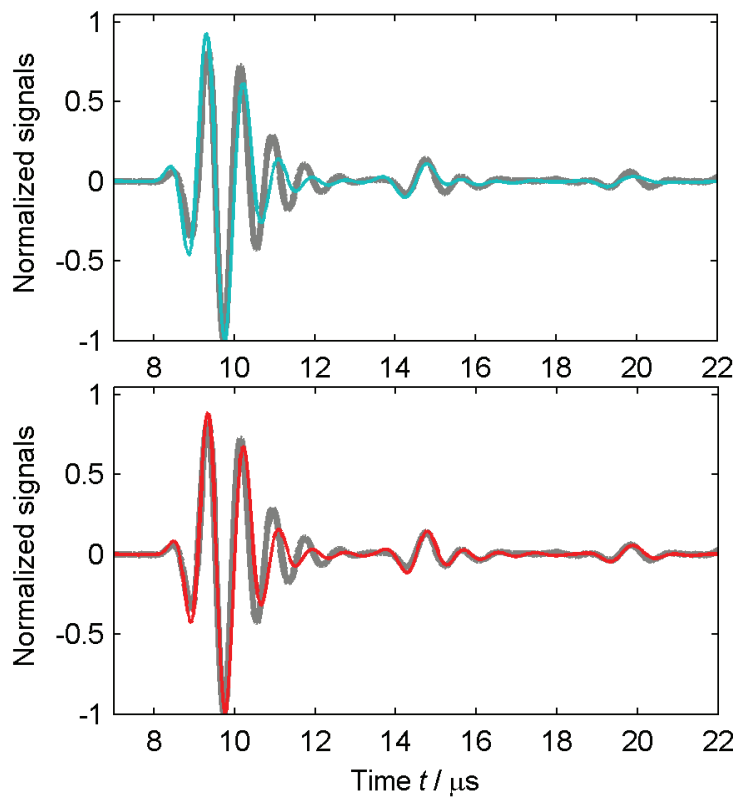

Fig. 9. Transient signals at the end of a PA 6 acoustic waveguide: measured (gray), simulated with the DMA data set (top, cyan) and with the inverse solution of the guided wave approach (bottom, red)

\section{Discussion}

Simulation and measurement are finally in good agreement for both cases. Actually the first signal group of the simulated signal is a little bit different from the measured one, especially in the signal decay. This is mainly due to an imperfect transducer model the authors use for simplicity. However, the succeeding signal groups are at the right position and have the right amplitudes. But the period length of the DMA data set signal is increasing too much. So the assumed damping in the analyzed frequency band was too high.

Therewith it is clear that a pure DMA is not suited to provide accurate data for ultrasonic simulations in the high frequency range. The opposite may not necessarily hold but could not be checked within this contribution. Fig. 10 again exemplifies the problem.

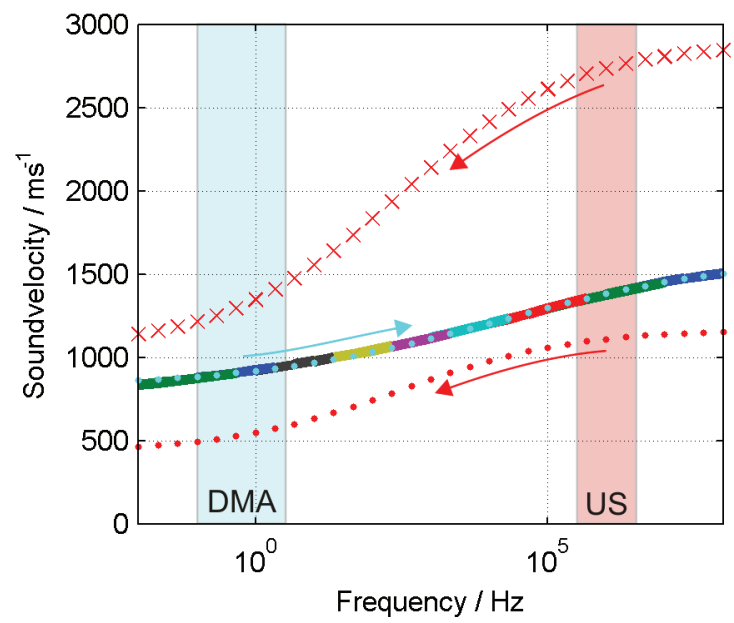

Fig. 10. Sound velocity dispersion calculated with the DMA and time temperature superposition (cyan) and with the transient ultrasonic method (red dotted: transversal wave; red crosses: longitudinal wave) the shadowed boxes visualize the frequency bands of the original measurements.

On the one hand there is a low frequency measurement which is used to extrapolate high frequency model parameters and on the other hand there is a high frequency measurement technique which is used to extrapolate low frequency material data. In both cases the assumed material model is the same. But as the DMA is only capable in providing effective moduli and the ultrasonic method provides separated values, the extrapolations cannot meet in the frequency band between.

If the material model should be parameterized in such a way that the calculated values are satisfactory in the entire frequency range it is necessary to use DMAs that provide separated mechanical moduli, too. Apart from that, more sophisticated time-temperature shifting algorithms have to be considered that additionally consider the modified density of the specimen if temperature is changed $[5,13]$.

\section{Conclusion}

With this contribution the DMA and guided ultrasonic waves were utilized to parameterize the fractional Zener model which is well suited to include viscoelastic material behavior in mechanical models. Both techniques are suited 
to give a good estimate of dispersion, especially the frequency dependent sound velocities for ultrasonic simulations, but the DMA needs additional parameterization in terms of longitudinal and transversal wave speeds to reach simulation results that are close to reality.

Actually it was not possible to combine the data in such a way that only one parameter set is sufficient to provide material parameters for the entire frequency range. Therefor more sophisticated DMAs are necessary.

\section{Acknowledgements}

The authors would like to thank Dr. Hauke Gravenkamp for supporting the SBFEM calculations, Christopher Schulte and Nadine Feldmann for their inspiring investigation of the DMA and the German Research Foundation (DFG) for financial support of the research project HE 2897/3-1 (determination of acoustic material properties).

\section{References}

[1] F. Mainardi, Fractional Calculus and Waves in Linear Viscoelasticity, Imperial College Press, London (2010); ISBN: 1-84816-329-0

[2] R. L. Bagley, P. J. Torvik, On the Fractional Calculus Model of Viscoelastic Behavior, Journal of Rheology 30(1),133-155 (1986); doi: 10.1122/1.549887

[3] F. Bause, A. Schröder, J. Rautenberg, B. Henning, H. Gravenkamp, Time-causal material modeling in the simulation of guided waves in circular viscoelastic waveguides, IEEE IUS, (2014); doi: 10.1109/ULTSYM.2014.0333

[4] A. J. Stavermann, F. Schwarzl, H.A. Stuart (ed.), Die Physik der Hochpolymeren, Springer, Heidelberg (1956)
[5] J. Mc Hugh, Ultrasound Technique for the Dynamic Mechanical Analysis (DMA) of Polymers, BAM-Dissertationsreihe 31 (2008)

[6] D. I. G. Jones, Handbook of Viscoelastic Vibration Damping, Wiley, NewYork (2001); ISBN: 978-0-471-49248-1

[7] S. S. S. Reddy, K. Balasubramaniam, C. V. Krishnamurthy, M. Shankar, Ultrasonic goniometry immersion techniques for the measurement of elastic moduli, Composite Structures 67, 3-17 (2005); doi: 10.1016/j.compstruct.2004.01.008

[8] J. Vishnuvardhan, C. V. Krishnamurthy, K. Balasubramaniam, Genetic algorithm reconstruction of orthotropic composite plate elastic constants from a single non-symmetric plane ultrasonic velocity data, Composites 38, 216-227 (2007); doi: 10.1016/j.compositesb.2006.06.006

[9] L. Bahr, M. Kaltenbacher, R. Lerch, Determination of damping and dispersion parameters with a combined measurement-simulation technique, IEEE Ultrasonics Symposium (2005); doi: 10.1109/ULTSYM.2005.1603189

[10] M. B. Reynolds, The Determination of the Elastic Constants of Metals by the Ultrasonic Pulse Technique, Trans. Am. Soc. for Metals 45, 839861 (1953)

[11] J. Rautenberg, F. Bause, B. Henning: Messsystem zur Bestimmung akustischer Kenngrößen stark absorbierender, transversal isotroper Kunststoffe, tm - Technisches Messen, 80(1), 28-37 (2013); doi: 10.1524/teme.2013.0005

[12] H. Gravenkamp, F. Bause, C. Song, On the computation of dispersion curves for axisymmetric elastic waveguides using the Scaled Boundary Finite Element Method, Computers and Structures, 131, 46-55 (2014); doi: 10.1016/j.compstruc.2013.10.014

[13] I. M. Ward, D. W. Hadley, An introduction to the mechanical properties of solid polymers. Wiley, Chichester (1993); ISBN: 0-471-93874-2 